\title{
Young Fields Demonstrate an Advanced State-of-the-Art
}

Presentations at the fourth European Quantum Electronics Conference (EQEC '93) held on 10-13 September 1993 in Florence was in essence dominated by the enormous progress and the advanced state-ofthe-art which has been achieved recently, not only in young fields such as atom optics and laser cooling, but also in well-established areas such as the generation of higher harmonics, optical parametric amplifiers and fibre lasers, to name but a few. This was also evident in the many lively discussions that took place during the poster sessions, which were as well attended as the sometimes crowded topical sessions.

The rich programme comprised 10 plenary talks in a single plenary session, 28 invited papers, and 267 contributed papers, including 186 posters. Owing to the satisfactorily large number of contributions, the oral presentations had to be held in either two or three parallel sessions covering the fields of laser device physics, laser spectroscopy, nonlinear processes, optical physics, quantum optics, ultrafast processes, and basic applications.

An interesting and topical review of the state-of-the-art and future development of solid-state lasers was given by A.I. Ferguson (Strathclyde) to open the meeting. Succeeding plenary lectures dealt with some of the most topical aspects in quantum electronics, quantum optics, nonlinear optics and cavity quantum electrodynamics in multi-quantum well devices, atom optics and atom interferometry, mesoscopic quantum coherence in cavity QED, electric dipole moments in atoms and neutrons, and applications of adaptive optics in astronomy. In particular, N. Ramsey (Harvard, USA) described how the latest determinations of the electric dipole moment of the neutron have been reconciled after initial apparent disagreement to give a value of $<9 \times 10^{-26} \mathrm{e} \mathrm{cm}$, but as the neutron sources used (in Grenoble and St. Petersburg) are out of operation it will be some time before the experiments continue. Meanwhile, a variety of experiments based on atomic physics have been taken up in electric dipole tests of time-reversal symmetry, but no nontime conserving effects have been identified so far. However, the most popular theory predicts a value that is $10^{3}$ times less than the present experimental limits. S. Haroche (Paris) described the transition from the quantum regime to the classical regime in the interaction of circular Rydberg atoms with the fields of a few photons in a high- $Q$ cavity using the measurement of their de Broglie wave phase shift in a Ramsey interferometer. Very impressive also was the demonstration of a new high sensitivity, low noise "infrared camera" by F. Capasso (AT\&T Bell Labs, Murray Hill, USA) based on a molecularbeam epitaxy lattice matched to a semi-insulating InP substrate consisting of $40 \mathrm{Si}$ doped $10.5 \mathrm{~nm}$ thick InGaAs quantum wells separated by $10 \mathrm{~nm}$ AllnAs barriers. The trick here is to make resonant structures in which there are bound states in a continuum (something
From the left, E. Picasso (Scuola Normale, Pisa), M. Inguscio, M. Petrone (Conference Secretary), and G.C. Righini.

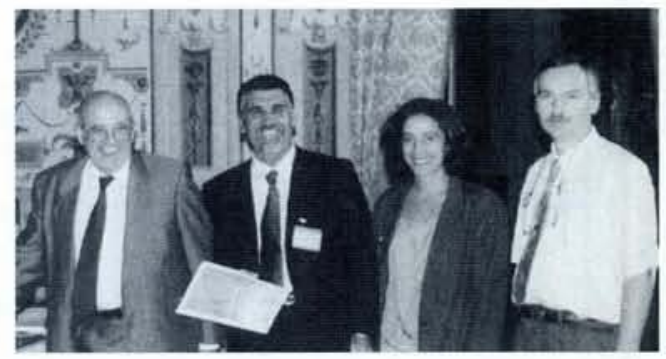

that was demonstrated to be theoretically possible in 1929) so that a non-linear current is generated as a result of three-photon interactions. Y. Yamamoto (Stanford, USA) discussed spontaneous emission arising from cavity-QED effects in multi-quantum well

devices. These devices are based on multilayer structures with a 3-5 $\mu \mathrm{m}$ in diameter hemispherical bump produced by reactive ion-beam etching. Preliminary results gave a coupling efficiency that is $50 \%$ less than the predicted level owing to inhomogeneous

\section{MONOLITHIC OPTICAL RESONATORS}

\section{Promising Alternatives for Squeezing Light}

There has been considerable progress recently in developing nonlinear optical resonators for frequency-conversion external to a laser cavity, and monolithic resonators are a particular implementation. They confine the resonated waves entirely to the nonlinear crystal, by an appropriate cut of the latter, and by dielectric mirrors deposited directly onto the crystal surfaces or by total internal reflection. The advantage of such designs lies in the low optical losses and the good mechanical stability of the cavity.

Present research is centred on efficient frequency doubling and on the development of resonators whose properties are tailored to quantum optical studies, e.g., the generation of squeezed light (whose amplitude fluctuations are reduced) by second-harmonic generation. Here, external nonlinear conversion has the advantage of being much simpler compared to processes internal to the laser cavity, since the dynamics of the laser medium play no rôle.

The principle of a MOTIRR with frequencyselective coupling prisms. The fundamental wave is coupled into the frequencydoubler using prism 1 while the harmonic wave is coupled out by frustrating the total internal reflection with prism 2. The harmonic output is optimised by adjusting the distance between the coupling prisms and the frequency-doubling crystal.

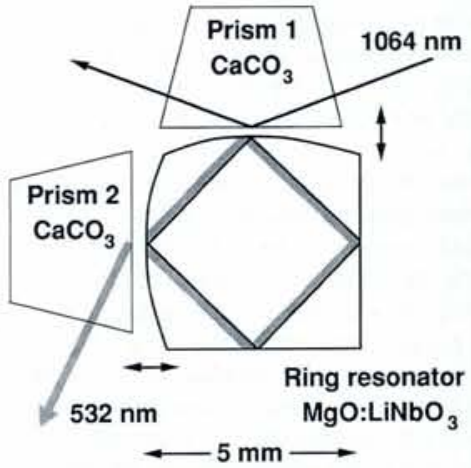

A doubly-resonant device, i.e., with high-reflectivity mirrors for both the laser and the harmonic optical frequencies, can be used to generate squeezed light at either the fundamental or the harmonic frequency by appropriate optimisation of the mirror reflectivities. We have recently demonstrated a quantum noise reduction of more than $50 \%$ at the fundamental frequency in a monolithic lithium-niobate standing-wave resonator with dielectric mirror coating [Kürz P. et al., Europhys. Lett., 24 (1993) 449]. An active frequency stabilisation of the monolithic resonator was achieved, thereby demonstrating the excellent potential for other applications. The latest developments show that singly resonant frequency-doubling can also be used to generate squeezed light (this has the advantage of an extremely simple implementation and excellent long-term stability). So monolithic frequency-doublers are promising alternatives to the optical parametric resonators usually used for this application.

Monolithic total internal reflection resonators (MOTIRR) that do not require dielectric coatings have recently been developed at Stanford University. These ring resonators are ideally suited for doubly-resonant harmonic generation because they resonate essentially all wavelengths of interest, have very low optical losses, and provide mechanically variable input-output coupling by frustrated total internal reflection. We have shown that such a device, with two coupling prisms for independent coupling of the fundamental and harmonic wave (see figure), gives highly efficient frequency-doubling (> $50 \%$ conversion efficiency) at input powers as low as $5 \mathrm{~mW}$ [Fiedler K. et al., Opt. Lett. 18 (Nov. 1993)] - a record efficiency for harmonic generation at low input powers. MOTIRRs are being studied for the generation of tunable radiation via parametric oscillation. It is likely that monolithic resonators will also be important for implementing quantum non-demolition measurement schemes. Finally, work in progress at Constance aims to generate a squeezed vacuum for possible application in gravitational wave interferometry using a monolithic, entirely solid-state, design. 
broadening. The advantages of using adaptive optics to improve drastically the resolution of modern optical telescopes were explained by F. Merkle (ESO, Garching).

Notable also was a report by E.O. Göbel (Marburg) of the observation, using femtosecond spectroscopy, of coherent coupling of free and bound excitonic transitions, as well as transitions arising from spatially separated quantum-well structures. There was also a first report (by W. Ertmer, Bonn) of the measurement of the scalar Aharonov-Bohm effect using atom interferometry with trapped neutral atoms. He presented as well first results for the diffraction of a metastable neon beam by a standing evanescent wave.
A morning session was devoted to a tribute to Veniamin Chebotayev by W.R. Bennett (Yale, USA), B. Cagnac (Paris), P. Franken (Tucson, USA), and K.M. Evenson (NIST, Boulder, USA).

EQEC is organized by the Quantum Electronics and Optics Division of EPS. It represents the main European forum for the growing number of European scientists wishing to discuss and present work concerned with the various aspects of quantum electronics, laser physics and its applications. The conference started to be held biennially in 1989 and this year it was combined with the 7th Italian Conference on Quantum Electronics

\section{FEMTOSECOND Ti: SAPPHIRE LASERS \\ New Workhorses for Ultrafast Spectroscopy}

The last few years have brought about significant advances in the physics and applications of ultrashort-pulse lasers. The recent appearance of ultrabroad-band, high quality Ti:sapphire laser crystals motivated the development of new ultrafast optical modulation techniques that are capable of shaping optical pulses of down to a few femtoseconds duration. The discovery of "self-mode-locking" by Spence et al. [Opt. Lett. 16 (1991) 42] and the exploitation of a "soliton-like" interplay between self-phase modulation and anomalous dispersion [see, e.g., Krausz F. et al., IEEE J. Quantum Electron. QE-28 (1992) 2097] represent major steps towards a powerful, new femtosecond laser technology based on solid-state gain materials.

The principle pulse-shaping effects in the self mode-locked Ti: sapphire laser are self focusing and self-phase modulation (SPM), both induced by the optical Kerr effect in the laser crystal. In combination with an intracavity aperture and negative group delay dispersion (GDD), they can result in efficient shortening and stabilisation of the modelocked pulse circulating in the cavity. The shortest achievable pulse duration is primarily determined by the bandwidth over which the negative intracavity GDD is nearly constant. Improving the dispersion properties of the intracavity prism pair, which has been the only low-loss source of broadband negative GDD until recently, led to a significant improvement in mode-locking performance. This work culminated in the development of quartz prism controlled Ti: sapphire lasers capable of generating optical pulses significantly less than $15 \mathrm{fs}$ in duration.

A schematic illustration of the mirror-dispersion controlled Ti:sapphire laser, and an interferometric autocorrelation trace of 11 fs pulses generated by the laser. The mirrors M1, M4 and M5 are the dispersive mirrors described in the text.

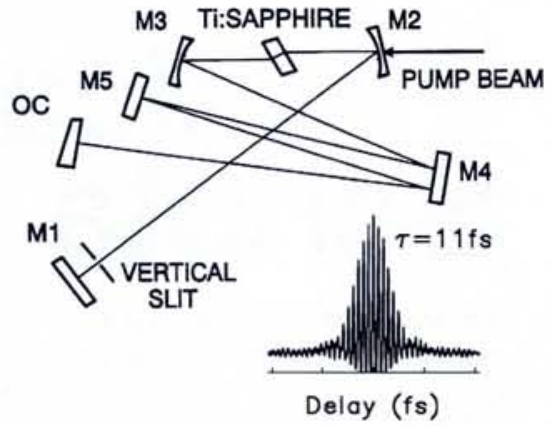

A new physical concept that can be exploited for broadband, essentially loss-free GDD-control uses multilayer dielectric mirrors. A "chirped" quasi-quarterwave dielectric coating is fabricated by modulating the multilayer period during the deposition process. The variation of the optical thickness of the layer gives a field penetration depth that depends on the optical frequency, as is the group delay which is experienced by different frequency components upon reflection at the mirror. Careful design ensures that a nearly constant negative GDD can be realised over a frequency range as broad as $\approx 80 \mathrm{THz}$ [Szipöcs R. et al., Opt. Lett., in press].

A Ti: sapphire laser which produces stable and reproducible pulses down to 11 fs in duration has been constructed using these mirrors. The absence of intracavity elements other than the gain medium and an aperture leads to unprecedented stability in the sub-20 fs regime [Stingl A., et al., Opt. Lett., in press]. Once mode-locked, the laser automatically delivers pulses in the $10-15$ fs range without any further optimisation owing to a cavity GDD that is completely insensitive to resonator alignment, in strong contrast with prism-controlled systems. These features make this mirror dispersioncontrolled Ti:sapphire femtosecond laser ideally suited for use as a front-end oscillator in complicated, femtosecond amplifier systems. The new technique also removes a constraint set by the minimum prism separation on the resonator length, and thus opens the way towards the development of high repetition rate, femtosecond self-mode-locked systems. Further improvements in engineering the cavity mirror dispersion will allow the development of a reliable sub-10 fs oscillator technology for the entire visible and near-infrared spectral range.

\section{A. Schmidt}

Abteilung Quantenelektronik und Lasertechnik, TU Wien
(EQUAP '93). Organized by M. Inguscio and G.C. Righini (Conference Co-Chairs) and J. Mlynek, with M. Inguscio as the Programme Co-Chair. The 362 participants from 25 countries included an encouragingly large number of students and young scientists. Despite the fact that the attractions in Florence represented strong competition, the level of participation remained remarkably good - maybe owing to the rewarding cultural events. Last but not least, Italian charm reconciled a few technical problems arising mainly due to the age of the venue - the impressive and picturesque Villa del Poggio Imperiale.

EQEC ' 94 will be held on 28 August - 2 September 1994 in parallel with the CLEO-Europe '94 at the RAI Congress Centre, Amsterdam. For information, contact: J. Mlynek, Chairman, EPS QEO Division, Fakultät für Physik, Universität Konstanz, Postfach 5560, D-78434 Constance (tel./fax: +49-7531-88 $3818 / 883072$ ).

\section{Letter to the Editor}

\section{Eur. J. Phys. Coverage}

I must ask for space to comment on the account, in the Europhysics News 25th EPS Anniversary issue, of the launching of the European Journal of Physics. The background your contributor paints for the journal is almost exclusively devoted to the activity of the EPS Advisory Committee on Physics Education but, as you are well aware, the journal was an enterprise embarked upon jointly by the EPS and The Institute of Physics in the UK. "The IOP had proposed to EPS that they publish jointly a new journal on physics education at university level", you report, and that EPS agreed the IOP's recommendation of myself as Editor-in-Chief (p. 151).

Now, I was not a party to the exchange of correspondence between the IOP and the EPS, but "a journal on physics education at university level" does not describe what I believed I was being asked to work on. After a number of years of service on the Editorial Boards of J. Phys. B and of Reports on Progress in Physics I had come to realise that the Institute had no vehicle for the publication of articles representing unconventional views on received topics in physics, for reflections, for alternative approaches: something other than primary research, on the one hand, or scholarly reviews, on the other. I made this point in correspondence with the Publications Committee of the Institute. When, some time later, I was informed of the discussions between the two bodies and that the points I had made might be incorporated in the subject coverage of a new journal, I felt honoured to be invited to act as Editor and accepted with enthusiasm. I believed that, since I was at that time an Individual Ordinary Member of the EPS (and have remained so ever since), the invitation was being made partly on account of my separate membership of the the two participating bodies.

The Agreement between the two bodies, made the 30th March 1979, included under "Title and Subject Coverage" the following statement: "The journal will publish articles in the following categories:

a) articles which are explicitly educational, that is, of a tutorial or pedagogical nature on particular topics in physics, or relating to methods of teaching physics as practised in 